\title{
Function-on-Function Regression with Mode-Sparsity Regularization
}

\author{
PEI YANG, South China University of Technology and Arizona State University \\ QI TAN, South China Normal University \\ JINGRUI HE, Arizona State University
}

\begin{abstract}
Functional data is ubiquitous in many domains, such as healthcare, social media, manufacturing process, sensor networks, and so on. The goal of function-on-function regression is to build a mapping from functional predictors to functional response. In this article, we propose a novel function-on-function regression model based on mode-sparsity regularization. The main idea is to represent the regression coefficient function between predictor and response as the double expansion of basis functions, and then use a mode-sparsity regularization to automatically filter out irrelevant basis functions for both predictors and responses. The proposed approach is further extended to the tensor version to accommodate multiple functional predictors. While allowing the dimensionality of the regression weight matrix or tensor to be relatively large, the modesparsity regularized model facilitates the multi-way shrinking of basis functions for each mode. The proposed mode-sparsity regularization covers a wide spectrum of sparse models for function-on-function regression. The resulting optimization problem is challenging due to the non-smooth property of the mode-sparsity regularization. We develop an efficient algorithm to solve the problem, which works in an iterative update fashion, and converges to the global optimum. Furthermore, we analyze the generalization performance of the proposed method and derive an upper bound for the consistency between the recovered function and the underlying true function. The effectiveness of the proposed approach is verified on benchmark functional datasets in various domains.
\end{abstract}

CCS Concepts: • Computing methodologies $\rightarrow$ Machine learning algorithms;

Additional Key Words and Phrases: Function-on-function regression, mode-sparsity regularization

\section{ACM Reference format:}

Pei Yang, Qi Tan, and Jingrui He. 2018. Function-on-Function Regression with Mode-Sparsity Regularization. ACM Trans. Knowl. Discov. Data. 12, 3, Article 36 (March 2018), 23 pages.

https://doi.org/10.1145/3178113

This work is supported by National Natural Science Foundation of China under Grant no. 61473123, Natural Science Foundation of Guangdong Province under Grant no. 2017A030313370, National Science Foundation under Grant No. IIS-1552654, and Grant no. CNS-1629888, the U.S. Department of Homeland Security under Grant Award Number 2017-ST-061-QA0001, and an IBM Faculty Award. The views and conclusions are those of the authors and should not be interpreted as representing the official policies of the funding agencies or the governments.

Authors' addresses: P. Yang, Department of Computer Science, South China University of Technology, Tianhe District, Guangzhou, China 510640; email: cs.pyang@gmail.com; Q. Tan, Department of Computer Science, South China Normal University, Tianhe District, Guangzhou, China 510630; email: tanqi@scnu.edu.cn; J. He, School of Computing, Informatics and Decision Systems Engineering, Arizona State University, Tempe, AZ, USA 85281; email: jingrui.he@gmail.com.

Permission to make digital or hard copies of all or part of this work for personal or classroom use is granted without fee provided that copies are not made or distributed for profit or commercial advantage and that copies bear this notice and the full citation on the first page. Copyrights for components of this work owned by others than ACM must be honored. Abstracting with credit is permitted. To copy otherwise, or republish, to post on servers or to redistribute to lists, requires prior specific permission and/or a fee. Request permissions from permissions@acm.org.

(C) 2018 ACM 1556-4681/2018/03-ART36 \$15.00

https://doi.org/10.1145/3178113 


\section{INTRODUCTION}

Functional data analysis (FDA) has been widely used in many areas, such as engineering, biomedicine, meteorology, economics, and so on, (Ramsay and Silverman 2005). In FDA, the ideal units of observation are functions defined on some continuous domains (e.g., time, spatial, imaging, spectral domains, and genomic locations), and the observed data consists of a sample of functions taken from some population sampled on a discrete grid (Morris 2015). For example, the mass spectrometry data (Koomen et al. 2005) contains mass spectra from a study to find serum proteomic markers for pancreatic cancer performed at the University of Texas MD Anderson Cancer Center. Each spectrum is a spiky function whose peaks correspond to proteins and peptides with molecular mass. Another example is neuroimaging, where the activation levels observed at voxels in the brain are the responses over the three dimensions of space and possibly time as well (Levitin et al. 2007). The distinctive characteristic of FDA is that it treats the infinite-dimensional data as real-valued functions rather than discrete, finite-dimensional vectors that are commonly used by existing data mining techniques.

In this article, we propose a novel Functional regression model with Mode-sparsity regularization (FarMost) for function-on-function regression, where both predictors and responses are functional data. As the name suggests, function-on-function regression aims to build a mapping between input functions (predictors) and output function (response). The main idea of FarMost is to represent the regression coefficients between the predictor and the response as the double expansion of basis functions, and then employ a mode-sparsity regularization on the regression weight matrix to truncate irrelevant basis functions. The intuition behind the proposed model is that we allow the dimensionality of the regression weight matrix to be relatively large to accommodate more basis functions for a potentially improved representation capability, while using the mode-sparsity regularization to automatically select the most informative basis functions. The mode-sparsity regularization enables the co-shrinking of basis functions for both predictors and responses. Meanwhile, it provides a flexible mechanism to adjust the sparsity of the regression weights, and covers a broad spectrum of sparse models for functional regression. Furthermore, we study the generalization performance of FarMost in the context of reconstructing the ground-truth function from the noisy observations. The theoretical analysis shows an upper bound regarding the discrepancy between the true underlying functional response and the recovered one.

The proposed FarMost model is further extended to its tensor version, allowing for the regression of functional response on multiple functional predictors. In this extension, the regression weights are in the tensor form in which each mode corresponds to either the response or one of the predictors. The mode-sparsity imposed on the regression weights facilitates the automatic truncation of basis functions for each mode.

The resulting optimization problem is convex but non-smooth due to the non-smoothness of the introduced mode-sparsity. To address this problem, we transform the non-smooth objective function into a smooth one by making use of an auxiliary function. Then, a simple and efficient algorithm is developed to solve the reformulated problem, which works in an iterative update fashion. The proposed algorithm is guaranteed to converge to the global optimum. The effectiveness of the proposed model is evaluated on the benchmark functional datasets collected from different domains, including weather data, electromyographical (EMG) data, and diffusion tensor imaging (DTI) data.

In summary, the main contributions of this article are as follows:

(1) We propose a novel function-on-function regression model FarMost based on modesparsity regularization that facilitates the multi-way selection of basis functions in building the regression model. 
(2) We develop an efficient algorithm to solve the optimization problem in an iterative update fashion, and prove its global convergence.

(3) We derive an upper bound for the consistency between the true underlying function and the recovered one.

(4) We extend FarMost to the tensor version to regress the response on multiple functional predictors.

(5) We demonstrate the effectiveness of FarMost on various functional datasets.

The rest of the article is organized as follows. After the review of the related work in Section 2, we present the proposed FarMost model in Section 3, and its tensor extension in Section 4. Section 5 shows the experimental results. Finally, we conclude the article in Section 6.

\section{RELATED WORK}

We review the related work on FDA (Ramsay and Silverman 2005). In particular, we focus on functional regression, which is one of the most active areas in FDA. According to the type of response, functional regression can be classified into either scalar-on-function regression or function-onfunction regression.

Most of the current functional regression work focus on scalar-on-function regression, which involves functional predictor and scalar response. To name a few, the Principal Analysis by Conditional Expectation (PACE) model aimed at estimating functional principal component scores for sparse longitudinal data (Yao et al. 2005a); the penalized functional regression method used truncated power series as basis functions to build the regression model with $\ell_{2}$ penalties (Goldsmith et al. 2011); the functional linear model adopted spline basis function for the regression penalized by roughness regularization (Zhang et al. 2007); the functional linear regression model introduced in Yuan and Cai (2010) is a general Reproducing Kernel Hilbert Space (RKHS) approach regularized by roughness penalty. In contrast, some work on the functional regression used sparsity constraints to build the model. For instance, the FLiRTI model (James et al. 2009) used $\ell_{1}$ penalty to encourage sparsity for interpretability; the Wavelet-based approach (Zhao et al. 2012, 2015) viewed the functional regression as a variable selection problem and used Lasso to identify the few important wavelet coefficients; the functional generalized linear model (Zhu and Cox 2009) applied a group Lasso penalty to select useful functional covariates among multiple curves; the sparse functional linear regression model (Lee and Park 2012) imposed different sparsity penalties such as Lasso and adaptive Lasso on the regression coefficients; the functional additive regression model (Fan et al. 2015) used group Lasso to perform variable selection across multiple functional predictors using orthogonal basis transform.

There has been comparatively less work on function-on-function regression, which involves both functional predictor and functional response (Morris 2015). The basic function-on-function regression model was introduced by Ramsay and Silverman (2005). Functional Principal Component Analysis (FPCA) is a popular technique used in functional regression model. For example, FPCreg (Yao et al. 2005b) is a non-parametric regression model based on functional principal component decomposition; the Functional Additive Models (FAM) (Müller and Yao 2008) utilized functional principal components in an additive way. Regularization is widely used in the function-onfunction model to avoid overfitting. In Harezlak et al. (2007), different regularization techniques, including basis truncation, roughness penalty, and sparsity penalty were adopted for linear Bspline basis functions. The penalized function-on-function regression (Ivanescu et al. 2015) accommodated multiple functional predictors and response observed on dense or sparse grids, and applied quadratic roughness penalties on the model. The functional additive mixed model (Scheipl et al. 2015) used quadratic penalty to build the regression model for functional response on both 
functional and scale predictor. The multi-level functional regression approach (Meyer et al. 2015) incorporated various basis expansions, including principal components, spline-based, and waveletbased functional representations into the regression model. The Dynamic Retrospective Functional Regression (DRFR) model (Gervini 2015) utilized time warping to characterize phase variability explicitly, and used roughness-penalty to control the smoothness. In addition, some non-linear functional regression models have been proposed, such as the triple-basis estimator (3BE) (Oliva et al. 2015) and the functional RKHS approach (Kadri et al. 2010, 2016).

In this article, we focus on building a function-on-function regression model by using modesparsity regularization for multi-way selection of basis functions, which is distinctive from the existing work on FDA. This article extends our previous work (Yang and He 2016) substantially by providing the theoretical analysis regarding the global convergence and the recovering consistency of the proposed approach, the tensor extension of FarMost, as well as the comprehensive empirical evaluations.

\section{THE PROPOSED FARMOST APPROACH}

We first present the FarMost model, and then introduce an iterative update algorithm to solve the resulting optimization problem. The generalization performance of the proposed model will be discussed at the end of this section.

\subsection{FarMost Model}

Let $\mathcal{S}$ and $\mathcal{T}$ be two interval domains. Denote the functional predictor by $x(s) \in \mathcal{R}^{n \times 1}$ measured at grid point $s \in \mathcal{S}$, and functional response by $y(t) \in \mathcal{R}^{n \times 1}$ measured at $t \in \mathcal{T}$, where $n$ is the number of curves. Note that we do not require the predictor and response to be defined on the same domain. Here, the goal is to build a regression model of functional response $y$ on functional predictor $x$.

Before diving into the details, we first introduce some notation. Let $\|W\|_{F}$ be the Frobenius norm of a matrix $W$. The $\ell_{p, q}$ norm of a matrix $W$ is defined as

$$
\|W\|_{p, q}=\left[\sum_{i}\left(\sum_{j}\left|W_{i j}\right|^{p}\right)^{q / p}\right]^{1 / q} .
$$

The dual norm of $\|W\|_{p, q}$ is denoted by $\|W\|_{p^{*}, q^{*}}$, where $\frac{1}{p}+\frac{1}{p^{*}}=1, \frac{1}{q}+\frac{1}{q^{*}}=1$, and $p, q \in[1, \infty]$. Let $x=\operatorname{vec}(X)$ be the vectorization of the matrix $X$ into a vector $x$. For two matrices $X$ and $Y$, let $X \odot Y$ and $X \otimes Y$ be the Hadamard product (or entrywise product) and Kronecker product, respectively. $\operatorname{diag}(x)$ returns a square diagonal matrix with the elements of vector $x$ on the main diagonal. Matricization is the process of reordering the elements of an $N$-way tensor into a matrix. The $i$-mode matricization of a tensor $\mathcal{W}$ (or a matrix $W$ ) is denoted by $\mathcal{W}_{(i)}\left(\right.$ or $\left.W_{(i)}\right)$. For example, for a matrix $W$ which is a two-way tensor, we have $W_{(1)}=W$ and $W_{(2)}=W^{T}$. The $i$-mode product of a tensor $\mathcal{W}$ with a matrix $X$ is denoted by $\mathcal{W} \times_{i} X$. Please refer to the survey paper of tensor for the details (Kolda and Bader 2009).

The basic functional regression model (Ramsay and Silverman 2005) can be represented as

$$
y(t)=\int_{\Omega_{t}} x(s) \beta(s, t) d s+\varepsilon(t),
$$

where $\beta(s, t)$ is a bivariate regression coefficient function, $\Omega_{t}$ is a $t$-dependent interval set, and $\varepsilon(t)$ is a residual function. Intuitively, the regression function $\beta(s, t)$ for a fixed value of $t$ can be interpreted as the related weight placed on $x(s)$ that is required to predict $y(t)$. 
We consider the bivariate regression function $\beta(s, t)$ as a double expansion in terms of $K_{1}$ basis functions $\varphi_{i}\left(1 \leq i \leq K_{1}\right)$ and $K_{2}$ basis functions $\theta_{j}\left(1 \leq j \leq K_{2}\right)$ such that

$$
\beta(s, t)=\sum_{i=1}^{K_{1}} \sum_{j=1}^{K_{2}} w_{i j} \varphi_{i}(s) \theta_{j}(t)=\varphi^{T}(s) W \theta(t),
$$

where $\varphi(s) \in \mathcal{R}^{K_{1} \times 1}, \theta(t) \in \mathcal{R}^{K_{2} \times 1}$, and $W$ is a $K_{1} \times K_{2}$ matrix of regression weight $w_{i j}$. By substituting this expansion, the basic regression model in Equation (1) becomes

$$
\begin{aligned}
y(t) & =\int_{\Omega_{t}} x(s) \varphi^{T}(s) W \theta(t) d s+\varepsilon(t) \\
& =X_{t} W \theta(t)+\varepsilon(t),
\end{aligned}
$$

where $X_{t}=\int_{\Omega_{t}} x(s) \varphi^{T}(s) d s$.

Next, we introduce the proposed functional regression model based on Mode-sparsity regularization (FarMost). The objective of FarMost is to simultaneously minimize the reconstruction loss resulting from the regression of the response on the predictor, and the mode-sparsity regularization imposed on the regression weights:

$$
\min _{W} \int\left\|y(t)-X_{t} W \theta(t)\right\|_{F}^{2} d t+\alpha_{1}\|W\|_{2, q}^{q}+\alpha_{2}\left\|W^{T}\right\|_{2, q}^{q}
$$

where $\alpha_{1}$ and $\alpha_{2}$ are non-negative parameters to control the importance of the sparsity regularizations. The mode-sparsity regularization imposes the row-wise sparisty on each mode-specific matricization of the two-way tensor $W$, i.e., $W_{(1)}$ and $W_{(2)}$. Specifically, the sparsity regularization $\|W\|_{2, q}^{q}$ encourages certain rows of $W$ to be sparse in order to filter out irrelevant basis functions from $\varphi$, while $\left\|W^{T}\right\|_{2, q}^{q}$ encourages certain columns of $W$ to be sparse to truncate irrelevant basis functions from $\theta$. Here, $q$ controls the degree of sparsity, where $1 \leq q \leq 2$. A smaller $q$ could lead to a more sparse $W$. In particular, $q=2$ corresponds to the squared Frobenius norm $\|W\|_{F}^{2}$, while $q=1$ corresponds to the $\ell_{2,1}$ norm $\|W\|_{2,1}$. The mode-sparsity regularization provides a wide spectrum of sparse models for function-on-function regression by varying the value of $q$. An illustrative example will be shown in the experiment section.

The intuition behind the proposed model is that we allow the dimensionality of $W$ to be relatively large to accommodate more basis functions contained in both $\varphi$ and $\theta$ for a potentially improved representation capability. Meanwhile, the mode-sparsity regularization is used to automatically and simultaneously pick out the most informative basis functions. In other words, the mode-sparsity regularization enables the joint selection of basis functions for both predictors and responses.

\subsection{Optimization}

Note that the objective function in Equation (4) is convex since both the loss function and $\ell_{2, q}$ norm are convex when $1 \leq q \leq 2$. However, the non-smoothness of $\ell_{2, q}$ norm $(1 \leq q<2)$ poses a considerable challenge on the optimization method. We will develop a simple and efficient algorithm to solve the problem.

We first introduce the following inequality (Nie et al. 2012), which will be used in the proof of convergence of the proposed algorithm.

Lemma 3.1. For any positive definite matrices $A, A_{t} \in \mathcal{R}^{m \times m}$, the following inequality holds when $0<p \leq 2$ :

$$
\operatorname{tr}\left(A^{\frac{p}{2}}\right)-\frac{p}{2} \operatorname{tr}\left(A A_{t}^{\frac{p-2}{2}}\right) \leq \operatorname{tr}\left(A_{t}^{\frac{p}{2}}\right)-\frac{p}{2} \operatorname{tr}\left(A_{t} A_{t}^{\frac{p-2}{2}}\right) .
$$


In Theorem 3.2, we transform the non-smooth objective defined in Equation (4) into a smooth one by making use of an auxiliary function, and derive an iterative update algorithm to solve the problem. A nice property of the proposed algorithm is its global convergence.

Theorem 3.2 (Update Rule). The objective in Equation (4) is strictly decreasing under the update

$$
W^{(k+1)}=\underset{W}{\arg \min } \int\left\|y(t)-X_{t} W \theta(t)\right\|_{F}^{2} d t+\sum_{i=1}^{2} \alpha_{i} \operatorname{tr}\left(W_{(i)}^{T} D_{i}^{(k)} W_{(i)}\right),
$$

until it reaches the optimum. $k$ is the iteration index, and $D_{i}^{(k)} \in \mathcal{R}^{K_{i} \times K_{i}}(1 \leq i \leq 2)$ is a diagonal matrix,

$$
D_{i}^{(k)}=\frac{q}{2}\left[\left(W_{(i)}^{(k)}\left(W_{(i)}^{(k)}\right)^{T}\right) \odot I\right]^{\frac{q-2}{2}}
$$

Proof. We make use of an auxiliary function to derive the update rule. Let $I$ be an identity matrix. Denote

$$
\begin{gathered}
\Phi_{i}=\left(W_{(i)} W_{(i)}^{T}\right) \odot I, \\
\Phi_{i}^{k}=\left(W_{(i)}^{(k)}\left(W_{(i)}^{(k)}\right)^{T}\right) \odot I .
\end{gathered}
$$

The objective function in Equation (4) is equivalently rewritten into

$$
\begin{aligned}
F(W) & =\int\left\|y(t)-X_{t} W \theta(t)\right\|_{F}^{2} d t+\sum_{i=1}^{2} \alpha_{i}\left\|W_{(i)}\right\|_{2, q}^{q} \\
& =\int\left\|y(t)-X_{t} W \theta(t)\right\|_{F}^{2} d t+\sum_{i=1}^{2} \alpha_{i} \operatorname{tr}\left(\Phi_{i}^{\frac{q}{2}}\right) .
\end{aligned}
$$

Next, the key issue is to design an auxiliary function for $F(W)$. Define a new function,

$$
G\left(W, W^{(k)}\right)=\int\left\|y(t)-X_{t} W \theta(t)\right\|_{F}^{2} d t+\sum_{i=1}^{2} \alpha_{i}\left[\frac{2-q}{2} \operatorname{tr}\left(\left(\Phi_{i}^{k}\right)^{\frac{q}{2}}\right)+\frac{q}{2} \operatorname{tr}\left(\Phi_{i}\left(\Phi_{i}^{k}\right)^{\frac{q-2}{2}}\right)\right] .
$$

Note that $G\left(W, W^{(k)}\right)$ is an auxiliary function of $F(W)$ due to the facts:

$$
G(W, W)=F(W)
$$

and

$$
G\left(W, W^{(k)}\right) \geq F(W)
$$

where the latter follows from Lemma 3.1 since both $\Phi_{i}$ and $\Phi_{i}^{k}$ are positive definite matrices.

Since $G\left(W, W^{(k)}\right)$ is an auxiliary function of $F(W), F(W)$ is non-increasing under the update

$$
W^{(k+1)}=\underset{W}{\arg \min } G\left(W, W^{(k)}\right) \text {. }
$$


Denote $J(W)=\int\left\|y(t)-X_{t} W \theta(t)\right\|_{F}^{2} d t$ for simplicity. Next, we obtain the derivative of $G\left(W, W^{(k)}\right)$ :

$$
\begin{aligned}
& \frac{\partial}{\partial W} G\left(W, W^{(k)}\right) \\
= & \frac{\partial}{\partial W} J(W)+\frac{\partial}{\partial W} \sum_{i=1}^{2} \alpha_{i} \operatorname{tr}\left[\Phi_{i} \frac{q}{2}\left(\Phi_{i}^{k}\right)^{\frac{q-2}{2}}\right] \\
= & \frac{\partial}{\partial W} J(W)+\frac{\partial}{\partial W} \sum_{i=1}^{2} \alpha_{i} \operatorname{tr}\left(\Phi_{i} D_{i}^{(k)}\right) \\
= & \frac{\partial}{\partial W} J(W)+\frac{\partial}{\partial W} \sum_{i=1}^{2} \alpha_{i} \operatorname{tr}\left[\left(\left(W_{(i)} W_{(i)}^{T}\right) \odot I\right) D_{i}^{(k)}\right] \\
= & \frac{\partial}{\partial W}\left[J(W)+\sum_{i=1}^{2} \alpha_{i} \operatorname{tr}\left(W_{(i)}^{T} D_{i}^{(k)} W_{(i)}\right)\right],
\end{aligned}
$$

where the last equality follows from the equivalence of the derivatives. Since

$$
F\left(W^{(k)}\right)=G\left(W^{(k)}, W^{(k)}\right) \geq \min _{W} G\left(W, W^{(k)}\right)=G\left(W^{(k+1)}, W^{(k)}\right) \geq F\left(W^{(k+1)}\right),
$$

the objective function $F(W)$ is non-increasing under the above update.

It is easy to verify that the auxiliary function $G\left(W, W^{(k)}\right)$ and the original function $F(W)$ have the same derivative at $W^{(k)}$, i.e.,

$$
\left.\nabla F(W)\right|_{W^{(k)}}=\left.\nabla G\left(W, W^{(k)}\right)\right|_{W^{(k)}} .
$$

Thus, if $W^{(k)}$ is a local optimal solution of $G\left(W, W^{(k)}\right)$, i.e., $\left.\nabla G\left(W, W^{(k)}\right)\right|_{W^{(k)}}=\mathbf{0}, W^{(k)}$ should be also the local optimal solution of $F(W)$ since

$$
\left.\nabla F(W)\right|_{W^{(k)}}=\left.\nabla G\left(W, W^{(k)}\right)\right|_{W^{(k)}}=0 .
$$

If $W^{(k)}$ is not a local optimal solution of $G\left(W, W^{(k)}\right)$, i.e., $W^{(k)} \neq W^{(k+1)}=\operatorname{argmin}_{W} G\left(W, W^{(k)}\right)$, the objective function $F(W)$ is strictly decreasing since

$$
F\left(W^{(k)}\right)=G\left(W^{(k)}, W^{(k)}\right)>\min _{W} G\left(W, W^{(k)}\right)=G\left(W^{(k+1)}, W^{(k)}\right) \geq F\left(W^{(k+1)}\right) .
$$

In summary, the objective function $F(W)$ is strictly decreasing under the update until it reaches the local optimum, i.e., $\left.\nabla F(W)\right|_{W^{(k)}}=0$.

In particular, for the special cases where $q=2$ and $q=1$, we have the following corollary, which can be easily derived from Theorem 3.2.

Corollary 3.3. If $q=2$ which corresponds to the squared Frobenius norm $\|W\|_{F}^{2}$, we have

$$
D_{i}^{(k)}=I \text {. }
$$

If $q=1$ which corresponds to the $\ell_{2,1}$ norm $\|W\|_{2,1}$, we have

$$
D_{i}^{(k)}=\operatorname{diag}\left(\frac{1}{2\left\|\left[W_{(i)}^{(k)}\right]_{1:}\right\|_{2}}, \ldots, \frac{1}{2 \|\left[W_{(i)}^{(k)}\right]_{K_{i}: \|_{2}}}\right)
$$

where $\left[W_{(i)}^{(k)}\right]_{j:}$ is the $j^{t h}\left(1 \leq j \leq K_{i}\right)$ row vector of $W_{(i)}^{(k)}$.

In Theorem 3.2, we prove the decrease of the objective function under the update. Furthermore, we can prove the convergence of the update algorithm, which is shown in Theorem 3.4. The 
proposed update algorithm is basically a second-order approximation method. But as shown in Theorem 3.2, our update rule is very simple.

Theorem 3.4 (Convergence). The proposed update algorithm is guaranteed to converge to the global optimum.

Proof. For the simplicity of illustration, we focus on the mode-sparsity regularization in objective Equation (4) only. But the conclusion holds for the whole objective. Denote $w=\operatorname{vec}\left(W_{(i)}^{T}\right)$. The mode-specific sparsity regularization with the vector variable $w$ is denoted by

$$
f(w)=\left\|W_{(i)}\right\|_{2, q}^{q} \text {. }
$$

Accordingly, the reformulated function with the vector variable $w$ is denoted by

$$
g(w)=\operatorname{tr}\left(W_{(i)}^{T} D_{i}^{(k)} W_{(i)}\right) .
$$

where $D_{i}^{(k)}$ is defined in Equation (6).

By Taylor's theorem, we have

$$
\begin{gathered}
f\left(w_{k+1}\right)=f\left(w_{k}\right)+p_{k}^{T} \nabla f\left(w_{k}\right)+\frac{1}{2} p_{k}^{T} \nabla^{2} f\left(w_{k}+\lambda_{1} p_{k}\right) p_{k}, \\
f\left(w_{k}\right)=f\left(w_{k+1}\right)-p_{k}^{T} \nabla f\left(w_{k+1}\right)+\frac{1}{2} p_{k}^{T} \nabla^{2} f\left(w_{k}+\lambda_{2} p_{k}\right) p_{k}, \\
g\left(w_{k+1}\right)=f\left(w_{k}\right)+p_{k}^{T} \nabla f\left(w_{k}\right)+\frac{1}{2} p_{k}^{T} \nabla^{2} g(w) p_{k},
\end{gathered}
$$

where $p_{k}=w_{k+1}-w_{k}$ is a search direction and $0<\lambda_{1}, \lambda_{2}<1$. Equation (11) follows from the facts: (1) $f\left(w_{k}\right)=g\left(w_{k}\right),(2) \nabla f\left(w_{k}\right)=\nabla g\left(w_{k}\right)$, and (3) $g(w)$ is a quadratic function. Therefore, $g(w)$ is a second-order approximation of $f(w)$ in the neighborhood of the point $w_{k}$. The zero-gradient condition for the $g(w)$ with respect to $p_{k}$ gives

$$
-\nabla f\left(w_{k}\right)=\nabla^{2} g(w) p_{k} .
$$

Then, we also have

$$
-p_{k}^{T} \nabla f\left(w_{k}\right)=p_{k}^{T} \nabla^{2} g(w) p_{k} .
$$

Denote the $r$ th row of $W_{(i)}$ by the row vector $u_{r}$ where $1 \leq r \leq K_{i}$. Let $Q_{r}$ be a diagonal matrix with a unique non-zero element $\left[Q_{r}\right]_{r r}=q(2-q)\left(u_{r} u_{r}^{T}\right)^{\frac{q}{2}-2}$. According to the definition of Hessian matrix, we have

$$
\nabla^{2} g(w)-\nabla^{2} f(w)=\sum_{r=1}^{K_{i}} Q_{r} \otimes\left(u_{r}^{T} u_{r}\right)
$$

Since both $Q_{r}$ and $u_{r}^{T} u_{r}$ are positive semi-definite, their Kronecker product $Q_{r} \otimes\left(u_{r}^{T} u_{r}\right)$ is positive semi-definite. Therefore, $\nabla^{2} g(w)-\nabla^{2} f(w)$ is also positive semi-definite. Then, we have

$$
p_{k}^{T} \nabla^{2} f\left(w_{k}+\lambda_{1} p_{k}\right) p_{k} \leq p_{k}^{T} \nabla^{2} g\left(w_{k}+\lambda_{1} p_{k}\right) p_{k}=-p_{k}^{T} \nabla f\left(w_{k}\right),
$$

and

Denote

$$
p_{k}^{T} \nabla^{2} f\left(w_{k}+\lambda_{2} p_{k}\right) p_{k} \leq p_{k}^{T} \nabla^{2} g\left(w_{k}+\lambda_{2} p_{k}\right) p_{k}=-p_{k}^{T} \nabla f\left(w_{k}\right) .
$$

and

$$
\frac{1}{2} p_{k}^{T} \nabla^{2} f\left(w_{k}+\lambda_{1} p_{k}\right) p_{k}=-\gamma_{1} p_{k}^{T} \nabla f\left(w_{k}\right)
$$

$$
\frac{1}{2} p_{k}^{T} \nabla^{2} f\left(w_{k}+\lambda_{2} p_{k}\right) p_{k}=-\gamma_{2} p_{k}^{T} \nabla f\left(w_{k}\right)
$$

where $0 \leq \gamma_{1}, \gamma_{2} \leq \frac{1}{2}$ 
According to Equations (9) and (10), we have

(i) if $\gamma_{1} \neq 0$ or $\gamma_{2} \neq 0$ :

$$
\begin{aligned}
& f\left(w_{k+1}\right)=f\left(w_{k}\right)+\left(1-\gamma_{1}\right) p_{k}^{T} \nabla f\left(w_{k}\right), \\
& p_{k}^{T} \nabla f\left(w_{k+1}\right)=\left(1-\gamma_{1}-\gamma_{2}\right) p_{k}^{T} \nabla f\left(w_{k}\right) .
\end{aligned}
$$

When $\gamma_{1}=\gamma_{2}=\frac{1}{2}$, we can set $0<c_{1}<\frac{1}{2}<c_{2}<1$; otherwise we can set $0<c_{1}<1-\gamma_{1}-\gamma_{2}<$ $c_{2}<1$ such that the proposed update algorithm meets the Wolfe conditions (Nocedal and Wright 2006):

$$
\begin{gathered}
f\left(w_{k+1}\right) \leq f\left(w_{k}\right)+c_{1} p_{k}^{T} \nabla f\left(w_{k}\right), \\
p_{k}^{T} \nabla f\left(w_{k+1}\right) \geq c_{2} p_{k}^{T} \nabla f\left(w_{k}\right) .
\end{gathered}
$$

Then, similar to Theorem 3.2 in Nocedal and Wright (2006), we can prove that our proposed algorithm converges to the local optimum.

ii) if $\gamma_{1}=\gamma_{2}=0$ :

Equations (12) and (13) are rewritten into

$$
\begin{gathered}
f\left(w_{k+1}\right)=f\left(w_{k}\right)+p_{k}^{T} \nabla f\left(w_{k}\right), \\
p_{k}^{T} \nabla f\left(w_{k+1}\right)=p_{k}^{T} \nabla f\left(w_{k}\right) .
\end{gathered}
$$

In this case, the function $f(w)$ degenerates as a hyperplane in the interval $\left[w_{k}, w_{k+1}\right]$. The search direction $p_{k}$ found by our proposed algorithm is the gradient descent direction. It directly arrives at the minimum of the hyperplane fragment $f(w)\left(w \in\left[w_{k}, w_{k+1}\right]\right)$.

For a convex function, the local optimum is also the global optimum (Nocedal and Wright 2006). Since the objective in Equation (4) is convex, the proposed update algorithm is guaranteed to converge to the global optimum.

We theoretically show the convergence of the proposed update method in Theorem 3.4. Furthermore, the empirical study conducted in the experiment section indicates that our algorithm converges fast and its performance becomes stable in a few iterations.

Note that Equation (5) is a quadratic optimization problem. We derive the closed-form solution for the regression weights $W$ as shown in Theorem 3.5.

Theorem 3.5 (Ортімum). The optimal solution for Equation (5) is as follows:

$$
\operatorname{vec}(W)=[A+D]^{-1} \operatorname{vec}(P),
$$

where

$$
\begin{gathered}
D=\alpha_{1} I_{K_{2}} \otimes D_{1}^{(k)}+\alpha_{2} D_{2}^{(k)} \otimes I_{K_{1}}, \\
A=\int\left[\theta(t) \theta^{T}(t)\right] \otimes\left(X_{t}^{T} X_{t}\right) d t . \\
P=\int X_{t}^{T} y(t) \theta^{T}(t) d t .
\end{gathered}
$$

Proof. The objective function in Equation (5) can be rewritten into

$$
\int\left\|y(t)-X_{t} W \theta(t)\right\|_{F}^{2} d t+\alpha_{1} \operatorname{tr}\left(W^{T} D_{1}^{(k)} W\right)+\alpha_{2} \operatorname{tr}\left(W D_{2}^{(k)} W^{T}\right) .
$$

The zero-gradient condition for the above function with respect to $W$ gives

$$
\int X_{t}^{T} X_{t} W \theta(t) \theta^{T}(t) d t+\alpha_{1} D_{1}^{(k)} W+\alpha_{2} W D_{2}^{(k)}=\int X_{t}^{T} y(t) \theta^{T}(t) d t .
$$




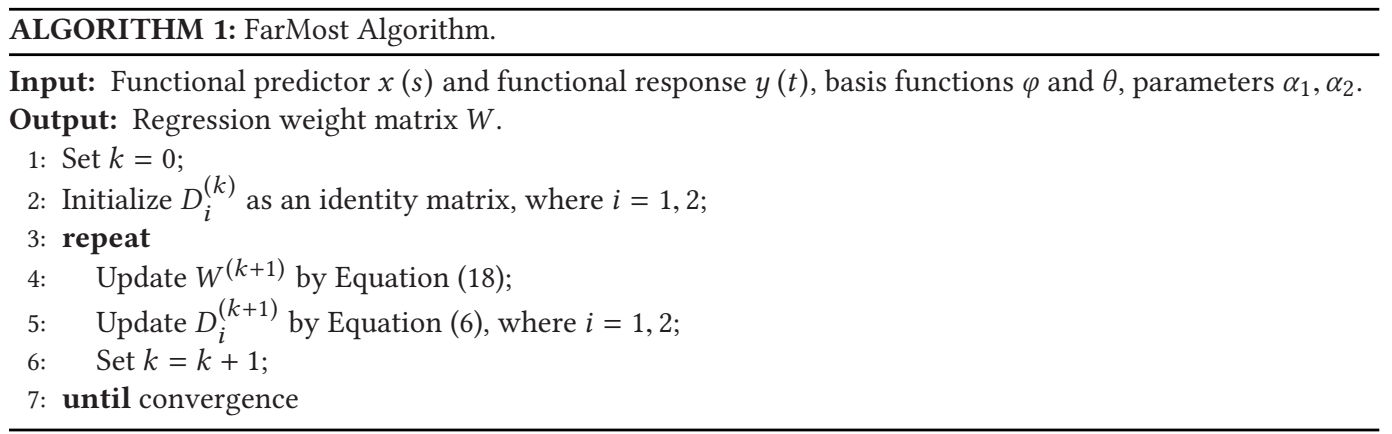

Based on the property of Kronecker product, vec $\left(U H V^{T}\right)=(V \otimes U) \operatorname{vec}(H)$ for any matrices $\mathrm{U}, \mathrm{V}$, and $\mathrm{H}$, we have

$$
\begin{aligned}
& \int \operatorname{vec}\left[X_{t}^{T} X_{t} W \theta(t) \theta^{T}(t)\right] d t+\operatorname{vec}\left[\alpha_{1} D_{1}^{(k)} W+\alpha_{2} W D_{2}^{(k)}\right]=\operatorname{vec}\left[\int X_{t}^{T} y(t) \theta^{T}(t) d t\right] \\
& \Rightarrow\left[\int\left(\theta(t) \theta^{T}(t)\right) \otimes\left(X_{t}^{T} X_{t}\right) d t\right] \cdot \operatorname{vec}(W)+\left[\alpha_{1} I_{K_{2}} \otimes D_{1}^{(k)}+\alpha_{2} D_{2}^{(k)} \otimes I_{K_{1}}\right] \cdot \operatorname{vec}(W)=\operatorname{vec}(P) \\
& \Rightarrow A \cdot \operatorname{vec}(W)+D \cdot \operatorname{vec}(W)=\operatorname{vec}(P) \\
& \Rightarrow \operatorname{vec}(W)=[A+D]^{-1} \cdot \operatorname{vec}(P),
\end{aligned}
$$

which completes the proof.

We summarize the above procedure into FarMost algorithm as shown in Algorithm 1. It iteratively updates the regression weight matrix $W$ and the diagonal matrix $D_{i}$ until convergence. Theorems 3.2 and 3.4 together demonstrate that the proposed FarMost algorithm is guaranteed to converge to the global optimum.

The most time or space consuming step in the FarMost algorithm is to update $W$ in Step 4. According to Equation (18), the size of the matrices involved in the computation is $K_{1}^{2} K_{2}^{2}$, where $K_{1}$ and $K_{2}$ are the numbers of basis functions. Note that even though the number of curves $n$ or the number of points $p$ is large, a far less number of basis functions would be enough to well represent the data. In other words, we usually have $K_{i} \ll \max (n, p)(i=1,2)$. Therefore, our method is scalable to large datasets. If $W$ is a large-size matrix, we can use conjugate gradient method (Golub and Loan 1996) to solve Equation (18). Also, the proposed FarMost algorithm is very simple, and enjoys the nice property of global convergence.

Note that some Quasi-Newton methods based on subgradient algorithms such as subBFGS (Yu et al. 2010) can deal with non-smooth problems. However, these methods have some limitations. First, to overcome the obstacle that the quasi-Newton direction at the non-smooth points is not necessarily a descent direction, they need to generalize multiple components of BFGS to subdifferentials, such as the local quadratic model, the identification of a descent direction, and the Wolf line search conditions. The resulting algorithm is relatively complex. Second, the optimal quasi-Newton direction is generally intractable due to the presence of a supremum over the entire subdifferential set.

\subsection{Special Cases}

The FarMost framework subsumes multiple basic functional regression models, such as the concurrent model, the historical model, and the global model (Ramsay and Silverman 2005). 
Consider that $\mathcal{S}$ and $\mathcal{T}$ are the same interval domains, i.e., $\mathcal{S}=\mathcal{T}$. Depending on the definition of $\Omega_{t}$ in Equation (1), the FarMost model can be instantiated to some special cases as follows:

(1) $\Omega_{t}=\{t\}$ : This corresponds to the concurrent model, indicating that $y(t)$ depends on $x(t)$ only;

(2) $\Omega_{t}=\{s \mid s \leq t, s \in \mathcal{S}\}$ : This corresponds to the historical model, indicating that the response is related to the historical data of the predictor;

(3) $\Omega_{t}=\mathcal{S}$ : This corresponds to the global model, indicating that the response depends on the whole data of the predictor.

Taking the global model, i.e., $\Omega_{t}=\mathcal{S}$, as an example, Equation (1) is specialized as

$$
y(t)=\int x(s) \beta(s, t) d s+\varepsilon(t)=X W \theta(t)+\varepsilon(t),
$$

where $X=\int x(s) \varphi^{T}(s) d s$.

For any functional data $u(t)$ and $v(t)$, define $J_{u v^{T}}=\int u(t) v^{T}(t) d t$. According to Theorem 3.5, it is easy to obtain the following corollary.

Corollary 3.6 (Optimum of Global Model). When $\Omega_{t}=\mathcal{S}$, the optimal solution for Equation (5) becomes,

$$
\operatorname{vec}(W)=\left[J_{\theta \theta^{T}} \otimes\left(X^{T} X\right)+D\right]^{-1} \cdot \operatorname{vec}\left(X^{T} J_{y \theta^{T}}\right) .
$$

\subsection{Generalization Performance}

We study the generalization performance of the proposed method in the context of recovering an underlying true function from the noisy measurements. Let $y^{*}(t)$ be the underlying true functional response, and the noisy version $y(t)$ is obtained as follows:

$$
y(t)=y^{*}(t)+\epsilon(t),
$$

where $\epsilon(t)$ is the noisy function. Let $\hat{y}(t)=X_{t} \hat{W} \theta(t)$ be the optimal estimator where

$$
\hat{W}=\underset{W}{\arg \min } \int\left\|y(t)-X_{t} W \theta(t)\right\|_{F}^{2} d t+\sum_{i=1}^{2} \alpha_{i}\left\|W_{(i)}\right\|_{2, q}^{q} .
$$

Suppose $r_{1}$ and $r_{2}$ are the numbers of non-zero rows in matrices $\left[\int X_{t}^{T}[y(t)-\hat{y}(t)] \theta^{T}(t) d t\right] \in$ $\mathcal{R}^{K_{1} \times K_{2}}$ and $\left[\int X_{t}^{T} \epsilon(t) \theta^{T}(t) d t\right] \in \mathcal{R}^{K_{1} \times K_{2}}$, respectively. Let $r=\max \left\{r_{1}, r_{2}\right\}$. Note that $r$ indicates the extent of row sparsity in the above matrices. In the following theorem, we explore the consistency between the optimal estimator and the underlying true functions, and uncover its relationship with the model parameters.

TheOREm 3.7 (CONSISTENCY). The consistency between the optimal estimator $\hat{y}(t)$ and the groundtruth function $y^{*}(t)$ satisfies the inequality

$$
\left\|\int X_{t}^{T}\left[\hat{y}(t)-y^{*}(t)\right] \theta^{T}(t) d t\right\|_{F} \leq \frac{q}{2} r^{\frac{q^{*}-2}{2 q^{*}}} \sum_{i=1}^{2} \alpha_{i}\left\|\hat{W}_{(i)}\right\|_{2, q}^{q-1}+r^{\frac{q^{*}-2}{2 q^{*}}} C_{\epsilon},
$$

where $C_{\epsilon}=\left\|\int X_{t}^{T} \epsilon(t) \theta^{T}(t) d t\right\|_{2, q^{*}}$ indicates the noise level.

If the noise level $C_{\epsilon}$ satisfies $C_{\epsilon} \leq \frac{q\left(\alpha_{1}+\alpha_{2}\right)}{2}$, the consistency inequality can be rewritten into

$$
\left\|\int X_{t}^{T}\left[\hat{y}(t)-y^{*}(t)\right] \theta^{T}(t) d t\right\|_{F} \leq \frac{q}{2} r^{\frac{q^{*}-2}{2 q^{*}}} \sum_{i=1}^{2} \alpha_{i}\left(1+\left\|\hat{W}_{(i)}\right\|_{2, q}^{q-1}\right),
$$


Proof. The zero subgradient condition of Equation (20) gives

$$
\int 2 X_{t}^{T}[y(t)-\hat{y}(t)] \theta^{T}(t) d t=\alpha_{1} q\|\hat{W}\|_{2, q}^{q-1} \Phi+\alpha_{2} q\left\|\hat{W}^{T}\right\|_{2, q}^{q-1} \Theta,
$$

where the subgradients $\Phi \in \partial\|W\|_{2, q}$ and $\Theta \in \partial\left\|W^{T}\right\|_{2, q} . \partial\|W\|_{2, q}$ and $\partial\left\|W^{T}\right\|_{2, q}$ are the subdifferentials. According to the dual norm property of subdifferentials (Watson 1992), we have $\|\Phi\|_{2, q^{*}} \leq 1$ and $\|\Theta\|_{2, q^{*}} \leq 1$, respectively. Therefore, we can obtain

$$
\begin{aligned}
& 2\left\|\int X_{t}^{T}[y(t)-\hat{y}(t)] \theta^{T}(t) d t\right\|_{2, q^{*}} \\
= & \left\|\alpha_{1} q\right\| \hat{W}\left\|_{2, q}^{q-1} \Phi+\alpha_{2} q\right\| \hat{W}^{T}\left\|_{2, q}^{q-1} \Theta\right\|_{2, q^{*}} \\
\leq & \alpha_{1} q\|\hat{W}\|_{2, q}^{q-1} \cdot\|\Phi\|_{2, q^{*}}+\alpha_{2} q\left\|\hat{W}^{T}\right\|_{2, q}^{q-1} \cdot\|\Theta\|_{2, q^{*}} \\
\leq & \alpha_{1} q\|\hat{W}\|_{2, q}^{q-1}+\alpha_{2} q\left\|\hat{W}^{T}\right\|_{2, q}^{q-1} .
\end{aligned}
$$

Suppose $u$ and $v$ are non-zero real numbers, $u<v$, and $a_{1}, \ldots, a_{m}$ are positive real numbers. The generalized mean inequality shows

$$
\left(\frac{1}{m} \sum_{i=1}^{m} a_{i}^{u}\right)^{\frac{1}{u}} \leq\left(\frac{1}{m} \sum_{i=1}^{m} a_{i}^{v}\right)^{\frac{1}{v}}
$$

Thus, for a matrix $A$ with $m$ rows, we have $\|A\|_{F} \leq m^{\frac{q^{*}-2}{2 q^{*}}}\|A\|_{2, q^{*}}$ where $q^{*} \in[2, \infty]$. Furthermore, if $A$ is sparse, we can leverage the sparsity to get a tighter result. Suppose $m_{r}$ is the number of non-zero rows in $A$. Then, we have $\|A\|_{F} \leq m_{r}^{\frac{q^{*}-2}{2 q^{*}}}\|A\|_{2, q^{*}}$.

Therefore, according to the generalized mean inequality, we obtain

$$
\begin{aligned}
& \left\|\int X_{t}^{T}[y(t)-\hat{y}(t)] \theta^{T}(t) d t\right\|_{F} \\
\leq & r_{1}^{\frac{q^{*}-2}{2 q^{*}}}\left\|\int X_{t}^{T}[y(t)-\hat{y}(t)] \theta^{T}(t) d t\right\|_{2, q^{*}} \\
\leq & \frac{q}{2} r_{1}^{\frac{q^{*}-2}{2 q^{*}}} \sum_{i=1}^{2} \alpha_{i}\left\|\hat{W}_{(i)}\right\|_{2, q}^{q-1} \leq \frac{q}{2} r^{\frac{q^{*}-2}{2 q^{*}}} \sum_{i=1}^{2} \alpha_{i}\left\|\hat{W}_{(i)}\right\|_{2, q}^{q-1} .
\end{aligned}
$$

Likewise, we have

$$
\left\|\int X_{t}^{T} \epsilon(t) \theta^{T}(t) d t\right\|_{F} \leq r_{2}^{\frac{q^{*}-2}{2 q^{*}}}\left\|\int X_{t}^{T} \epsilon(t) \theta^{T}(t) d t\right\|_{2, q^{*}} \leq r^{\frac{q^{*}-2}{2 q^{*}}} C_{\epsilon} .
$$

By the triangular inequality, we have

$$
\begin{aligned}
& y(t)=y^{*}(t)+\epsilon(t) \\
\Rightarrow & \hat{y}(t)-y^{*}(t)=\hat{y}(t)-y(t)+\epsilon(t) \\
\Rightarrow & X_{t}^{T}\left[\hat{y}(t)-y^{*}(t)\right] \theta^{T}(t)=X_{t}^{T}[\hat{y}(t)-y(t)] \theta^{T}(t)+X_{t}^{T} \epsilon(t) \theta^{T}(t) \\
\Rightarrow & \int X_{t}^{T}\left[\hat{y}(t)-y^{*}(t)\right] \theta^{T}(t) d t=\int X_{t}^{T}[\hat{y}(t)-y(t)] \theta^{T}(t) d t+\int X_{t}^{T} \epsilon(t) \theta^{T}(t) d t
\end{aligned}
$$




$$
\Rightarrow\left\|\int X_{t}^{T}\left[\hat{y}(t)-y^{*}(t)\right] \theta^{T}(t) d t\right\|_{F} \leq\left\|\int X_{t}^{T}[\hat{y}(t)-y(t)] \theta^{T}(t) d t\right\|_{F}+\left\|\int X_{t}^{T} \epsilon(t) \theta^{T}(t) d t\right\|_{F} .
$$

Then, we have

$$
\left\|\int X_{t}^{T}\left[\hat{y}(t)-y^{*}(t)\right] \theta^{T}(t) d t\right\|_{F} \leq \frac{q}{2} r^{\frac{q^{*}-2}{2 q^{*}}} \sum_{i=1}^{2} \alpha_{i}\left\|\hat{W}_{(i)}\right\|_{2, q}^{q-1}+r^{\frac{q^{*}-2}{2 q^{*}}} C_{\epsilon} .
$$

If the noise level $C_{\epsilon}$ satisfies $C_{\epsilon} \leq \frac{q\left(\alpha_{1}+\alpha_{2}\right)}{2}$, we have

$$
\left\|\int X_{t}^{T}\left[\hat{y}(t)-y^{*}(t)\right] \theta^{T}(t) d t\right\|_{F} \leq \frac{q}{2} r^{\frac{q^{*}-2}{2 q^{*}}} \sum_{i=1}^{2} \alpha_{i}\left(1+\left\|\hat{W}_{(i)}\right\|_{2, q}^{q-1}\right),
$$

which completes the proof.

Theorem 3.7 shows the upper bound regarding the discrepancy between the underlying true function $y^{*}(t)$ and the recovered function $\hat{y}(t)$. From Equation (21), we can see that the upper bound is composed of two parts. The first part is related to the row sparsity of the regression weight matrix, while the second part is related to the noise level. It suggests that imposing the modesparsity regularization on the regression weights could reduce the upper bound for the recovering consistency.

According to Theorem 3.7, we have the following corollary.

Corollary 3.8. When using the $\ell_{2,1}$ norm (i.e., $q=1$ ), the consistency between the optimal estimator $\hat{y}(t)$ and the ground-truth function $y^{*}(t)$ satisfies the inequality

$$
\left\|\int X_{t}^{T}\left[\hat{y}(t)-y^{*}(t)\right] \theta^{T}(t) d t\right\|_{F} \leq \sqrt{r} \frac{\left(\alpha_{1}+\alpha_{2}\right)}{2}+\sqrt{r} C_{\epsilon} .
$$

If the noise level $C_{\epsilon}$ satisfies $C_{\epsilon} \leq \frac{\left(\alpha_{1}+\alpha_{2}\right)}{2}$, we have

$$
\left\|\int X_{t}^{T}\left[\hat{y}(t)-y^{*}(t)\right] \theta^{T}(t) d t\right\|_{F} \leq\left(\alpha_{1}+\alpha_{2}\right) \sqrt{r} .
$$

For FarMost with $\ell_{2,1}$ regularization, Corollary 3.8 provides the insights into what factors will affect the recovering consistency. It shows that the consistency between the optimal estimator and the underlying true function is related to the number of non-zero rows in the corresponding matrices, the noise level, and the tradeoff parameters $\alpha_{1}$ and $\alpha_{2}$. First, the sparsity regularization imposed on the model is likely to result in the smaller $r$, leading to the better recovered function $\hat{y}(t)$ which is more consistent with the true one $y^{*}(t)$. Second, when the noise goes to zero $(\epsilon(t) \rightarrow$ $0)$, the discrepancy between the recovered function and the true underlying one, i.e., $\hat{y}(t)-y^{*}(t)$, approaches zero since $\alpha_{1}+\alpha_{2}$ shrinks to zero. Third, even though the noise does not approach zero, the lower level of noise could lead to the better consistency between the recovered function and the true one.

\section{TENSOR EXTENSION OF FARMOST}

In this section, we extend FarMost to its tensor version to incorporate multiple functional predictors into building the regression model.

Without loss of generality, suppose we have one functional response $y(t) \in \mathcal{R}^{n \times 1}$ and two functional predictors, $x(s) \in \mathcal{R}^{n \times 1}$ and $z(r) \in \mathcal{R}^{n \times 1}$, where $n$ is the number of curves. Let $c$ be the index of the $c^{t h}(1 \leq c \leq n)$ curve. The basic functional regression model is represented as

$$
y_{c}(t)=\iint z_{c}(r) x_{c}(s) \beta(r, s, t) d r d s+\varepsilon(t) .
$$


We consider the multivariate regression function $\beta(r, s, t)$ as the multiple expansions in terms of $K_{1}$ basis functions $\rho_{i}\left(1 \leq i \leq K_{1}\right), K_{2}$ basis functions $\varphi_{j}\left(1 \leq j \leq K_{2}\right)$, and $K_{3}$ basis functions $\theta_{k}$ $\left(1 \leq k \leq K_{3}\right)$ such that

$$
\beta(r, s, t)=\sum_{i=1}^{K_{1}} \sum_{j=1}^{K_{2}} \sum_{k=1}^{K_{3}} w_{i j k} \rho_{i}(r) \varphi_{j}(s) \theta_{k}(t)=\mathcal{W} \times{ }_{1} \rho^{T}(r) \times_{2} \varphi^{T}(s) \times_{3} \theta^{T}(t),
$$

where $\rho(r) \in \mathcal{R}^{K_{1} \times 1}, \varphi(s) \in \mathcal{R}^{K_{2} \times 1}, \theta(t) \in \mathcal{R}^{K_{3} \times 1}$, and $\mathcal{W} \in \mathcal{R}^{K_{1} \times K_{2} \times K_{3}}$ is a three-way tensor of regression weight $w_{i j k}$. By substituting this expansion, Equation (25) becomes

$$
\begin{aligned}
y_{c}(t) & =\iint z_{c}(r) x_{c}(s) \beta(r, s, t) d r d s+\varepsilon(t) \\
& =\iint z_{c}(r) x_{c}(s)\left[\mathcal{W} \times{ }_{1} \rho^{T}(r) \times{ }_{2} \varphi^{T}(s) \times{ }_{3} \theta^{T}(t)\right] d r d s+\varepsilon(t) \\
& =\mathcal{W} \times \times_{1}\left[\int z_{c}(r) \rho^{T}(r) d r\right] \times{ }_{2}\left[\int x_{c}(s) \varphi^{T}(s) d s\right] \times{ }_{3} \theta^{T}(t)+\varepsilon(t) \\
& =\mathcal{W} \times{ }_{1} Z_{c} \times{ }_{2} X_{c} \times{ }_{3} \theta^{T}(t)+\varepsilon(t),
\end{aligned}
$$

where $X_{c}=\int x_{c}(s) \varphi^{T}(s) d s$ and $Z_{c}=\int z_{c}(r) \rho^{T}(r) d r$.

According to the property of mode products of a $d$-way tensor $\mathcal{W}$ with matrices $A_{i}(1 \leq i \leq d)$,

$$
\mathcal{Y}=\mathcal{W} \times{ }_{1} A_{1} \times_{2} A_{2} \cdots \times{ }_{d} A_{d} \Leftrightarrow \mathcal{Y}_{(d)}=A_{d} \mathcal{W}_{(d)}\left(A_{d-1} \otimes \cdots \otimes A_{1}\right)^{T},
$$

Equation (27) can be rewritten into

$$
y_{c}(t)=\theta^{T}(t) \mathcal{W}_{(d)} B_{c}^{T}=B_{c} \mathcal{W}_{(d)}^{T} \theta(t),
$$

where $B_{c}=X_{c} \otimes Z_{c}$ and $d=3$. Note that $B_{c} \in \mathcal{R}^{1 \times K_{1} K_{2}}$ and $\mathcal{W}_{(d)} \in \mathcal{R}^{K_{3} \times K_{1} K_{2}}$. Let $B \in \mathcal{R}^{n \times K_{1} K_{2}}$ be a matrix whose $c t h$ row vector is $B_{c}$. Then, based on Equation (28), we have

$$
y(t)=B \mathcal{W}_{(d)}^{T} \theta(t) .
$$

For the tensor version of FarMost, the objective is to minimize the reconstruction loss resulting from the regression of the response on the multiple predictors, as well as the mode-sparsity regularization on the regression weights:

$$
\min _{\mathcal{W}} \int\left\|y(t)-B \mathcal{W}_{(d)}^{T} \theta(t)\right\|_{F}^{2} d t+\sum_{i=1}^{d} \alpha_{i}\left\|\mathcal{W}_{(i)}\right\|_{2, q}^{q},
$$

where $\alpha_{i}(1 \leq i \leq d)$ is a non-negative parameter to control the importance of mode-sparsity regularization. The mode-specific sparsity $\left\|\mathcal{W}_{(i)}\right\|_{2, q}^{q}$ encourages certain rows of $\mathcal{W}_{(i)}$ to be sparse. In other words, it encourages certain slices of $\mathcal{W}$ along the $i$ th dimension to be sparse. In total, the mode-sparsity regularization $\sum_{i} \alpha_{i}\left\|\mathcal{W}_{(i)}\right\|_{2, q}^{q}$ allows the model to automatically and simultaneously select the most informative basis functions from each mode, while truncating the irrelevant ones. In other words, the mode-sparsity facilitates the multi-way selection of basis functions.

Note that the proposed model can be easily extended to incorporate multiple functional predictors by modeling them into a higher order $(d>3)$ tensor. Also, when $d=2$, it is reduced to the two-way model proposed in Section 3.

Similar to Theorem 3.2, we derive an update rule for the tensor version of FarMost in Theorem 4.1, and prove its global convergence. The proof of Theorem 4.1 is omitted for brevity. 
Theorem 4.1 (Update Rule). The objective in Equation (30) is strictly decreasing under the update,

$$
\mathcal{W}^{(k+1)}=\underset{\mathcal{W}}{\arg \min } \int\left\|y(t)-B \mathcal{W}_{(d)}^{T} \theta(t)\right\|_{F}^{2} d t+\sum_{i=1}^{d} \alpha_{i} \operatorname{tr}\left(\mathcal{W}_{(i)}^{T} D_{i}^{(k)} \mathcal{W}_{(i)}\right),
$$

until it reaches the optimum. $k$ is the iteration index, and $D_{i}^{(k)} \in \mathcal{R}^{K_{i} \times K_{i}}(1 \leq i \leq d)$ is a diagonal matrix,

$$
D_{i}^{(k)}=\frac{q}{2}\left[\left(\mathcal{W}_{(i)}^{(k)}\left(\mathcal{W}_{(i)}^{(k)}\right)^{T}\right) \odot I\right]^{\frac{q-2}{2}} .
$$

The next theorem shows the analytical solution for the regression weights in tensor form.

Theorem 4.2 (Ортімuм). The optimal solution for Equation (31) is as follows:

$$
\operatorname{vec}\left(\mathcal{W}_{(d)}\right)=\left[\left(B^{T} B\right) \otimes J_{\theta \theta^{T}}+D\right]^{-1} \cdot \operatorname{vec}\left(J_{\theta y^{T}} B\right),
$$

where $D$ is a diagonal matrix,

$$
D=\operatorname{diag}\left[\operatorname{vec}\left(\mathcal{P}_{(d)}\right)\right],
$$

where $\mathcal{P} \in \mathcal{R}^{K_{1} \times K_{2} \times K_{3}}$ is a three-way tensor whose entry is

$$
\mathcal{P}_{i j v}=\alpha_{1}\left[D_{1}^{(k)}\right]_{i i}+\alpha_{2}\left[D_{2}^{(k)}\right]_{j j}+\alpha_{3}\left[D_{3}^{(k)}\right]_{v v},
$$

for $1 \leq i \leq K_{1}, 1 \leq j \leq K_{2}$, and $1 \leq v \leq K_{3}$.

Proof. The objective function in Equation (31) can be rewritten into,

$$
\begin{aligned}
& \min _{\mathcal{W}} \int\left\|y(t)-B \mathcal{W}_{(d)}^{T} \theta(t)\right\|_{F}^{2} d t+\sum_{i=1}^{d} \alpha_{i} \operatorname{tr}\left(\mathcal{W}_{(i)}^{T} D_{i}^{(k)} \mathcal{W}_{(i)}\right) \\
= & \min _{W} \operatorname{tr}\left[\mathcal{W}_{(d)}^{T} J_{\theta \theta^{T}} \mathcal{W}_{(d)} B^{T} B-2 \mathcal{W}_{(d)}^{T} J_{\theta y^{T}} B+J_{y y^{T}}\right]+\sum_{i=1}^{d} \alpha_{i} \operatorname{tr}\left(\mathcal{W}_{(i)}^{T} D_{i}^{(k)} \mathcal{W}_{(i)}\right) .
\end{aligned}
$$

The zero-gradient condition of the above equation with respect to $\mathcal{W}_{(d)}$ gives

$$
J_{\theta \theta^{T}} \mathcal{W}_{(d)} B^{T} B+\mathcal{P}_{(d)} \odot \mathcal{W}_{(d)}=J_{\theta y^{T}} B,
$$

Based on the property of Kronecker product, $\operatorname{vec}\left(U H V^{T}\right)=(V \otimes U) \operatorname{vec}(H)$, for any matrices $\mathrm{U}$, $\mathrm{V}$, and $\mathrm{H}$, we have

$$
\begin{aligned}
& {\left[\left(B^{T} B\right) \otimes J_{\theta \theta^{T}}\right] \cdot \operatorname{vec}\left(\mathcal{W}_{(d)}\right)+\operatorname{vec}\left(\mathcal{P}_{(d)}\right) \odot \operatorname{vec}\left(\mathcal{W}_{(d)}\right)=\operatorname{vec}\left(J_{\theta y^{T}} B\right)} \\
& \Leftrightarrow\left[\left(B^{T} B\right) \otimes J_{\theta \theta^{T}}\right] \cdot \operatorname{vec}\left(\mathcal{W}_{(d)}\right)+\operatorname{diag}\left[\operatorname{vec}\left(\mathcal{P}_{(d)}\right)\right] \cdot \operatorname{vec}\left(\mathcal{W}_{(d)}\right)=\operatorname{vec}\left(J_{\theta y^{T}} B\right) .
\end{aligned}
$$

Therefore, optimizing Equation (31) is equivalent to solving the linear equations

$$
\left[\left(B^{T} B\right) \otimes J_{\theta \theta^{T}}+D\right] \cdot \operatorname{vec}\left(\mathcal{W}_{(d)}\right)=\operatorname{vec}\left(J_{\theta y^{T}} B\right),
$$

which completes the proof.

If $\mathcal{W}$ is a large-size tensor, the computation of inverse matrix is inefficient and space-consuming. Instead, we solve the linear equations in Equation (34) by using conjugate gradient method (Golub and Loan 1996). Also, in order to save the space, we just need to store the two matrices, $B^{T} B$ and $J_{\theta \theta^{T}}$, which is much smaller than their Kronecker product.

\section{EXPERIMENTS}

We compare the proposed FarMost method with different functional regression methods on three benchmark functional datasets collected from various domains. 
Table 1. Statistics of Different Datasets

\begin{tabular}{l|ccccc}
\hline Dataset & $n$ & $x$ & $|\mathcal{S}|$ & $y$ & $|\mathcal{T}|$ \\
\hline Weather & 35 & Temperature & 365 & Precipitation & 365 \\
LipEMG & 32 & EMG & 501 & Acceleration & 501 \\
DTI2 & 340 & FA-RCTS & 55 & FA-CCA & 93 \\
\hline
\end{tabular}

\subsection{Datasets}

The Canadian weather dataset was introduced in Ramsay and Silverman (2005) as one of their main examples of functional data. For 35 weather stations, the daily temperature and precipitation were averaged over a period of 30 years. Thus, there are 365 time points for either daily temperature or precipitation profile. The goal is to predict the log daily precipitation profile of a weather station from information on the daily temperature profile.

The LipEMG data ${ }^{1}$ consists of 32 records of the movement acceleration of the lower lip when a subject was repeatedly required to say the syllable "bob," embedded in the sentence, "Say bob again," and the corresponding EMG activities of the primary muscle depressing the lower lip, the depressor labii inferior

(Ramsay and Silverman 2002). Each of these measurements has 501 time points. According to Newton's second law, acceleration reflects the force applied to tissue by muscle contraction. Here, the goal is to predict the lip acceleration from the EMG activities.

The diffusion tensor imaging (DTI2) data was collected at Johns Hopkins University and the Kennedy-Krieger Institute (Basser et al. 2000). The dataset involves 340 DTI scans from subjects with multiple sclerosis (MS). MS is a demyelinating autoimmune-mediated disease that is associated with brain lesions and results in severe disability. From each DTI scan, fractional anisotropy (FA) profiles are obtained along the corpus callosum (FA-CCA) and right corticospinal (FA-RCTS) tracts. Each of the FA-CCA or FA-RCTS tracts has 93 or 55 grid points, respectively. The goal is to build a functional regression model to predict FA-CCA from FA-RCTS in order to study the spatial association between demyelinations along the FA-CCA and FA-RCTS tracts.

Table 1 shows the statistics of the datasets, where $|\mathcal{S}|$ and $|\mathcal{T}|$ are numbers of points in the corresponding domains.

The common pre-processing step in FDA is to convert discrete measured values to continuous functions by using the basis functions (Ramsay and Silverman 2005). In our experiments, we use Fourier series to fit the functional predictors and responses, respectively, which is smoothed via roughness penalty. Specifically, we use the "data2fd" function provided in FDA package ${ }^{2}$ to convert the data, and empirically set the parameter $\gamma=0.1$, which is used to control the importance of the roughness regularization.

\subsection{Evaluation Metrics and Comparison Methods}

We adopt different performance metrics to evaluate the proposed method in comparison with various functional regression algorithms.

Let $\hat{y}(t)=X_{t} \hat{W} \theta(t)$ be the predicted response function. The predicted and ground-truth regression functions for the $\operatorname{cth}(c=1, \ldots, m)$ curve are denoted by $\hat{y}_{c}(t)$ and $y_{c}(t)$, respectively, where $m$ is the number curves in the test data. RSS is the sum of squared residuals integrated over the

\footnotetext{
${ }^{1}$ http://www.stats.ox.ac.uk/ silverma/fdacasebook/lipemg.html.

${ }^{2}$ http://www.psych.mcgill.ca/misc/fda/.
} 


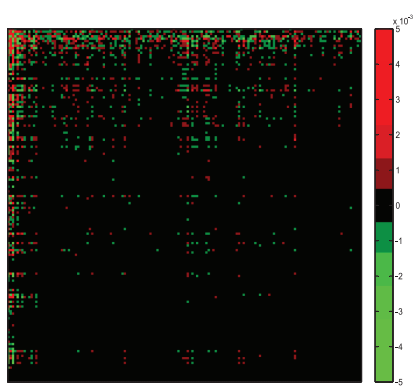

(a) $q=1.0$

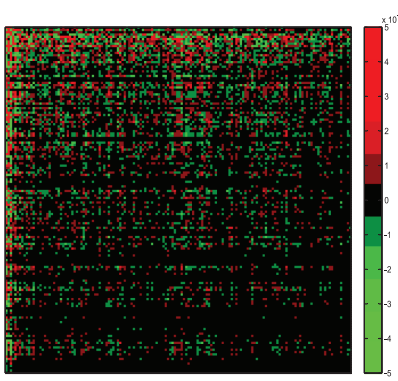

(b) $q=1.2$

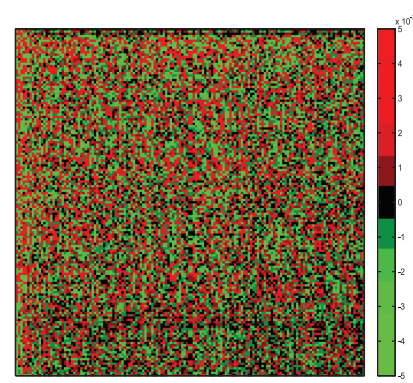

(c) $q=2.0$

Fig. 1. The Heatmaps of $W$. The point in black represents the zero entry in the matrix $W$. The matrix $W$ becomes sparser along with the decreased $q$ value.

domain, and MRSS is the mean of RSS over testing curves, defined as

$$
\text { MRSS }=\frac{1}{m} \sum_{c=1}^{m} \int\left[y_{c}(t)-\hat{y}_{c}(t)\right]^{2} d t .
$$

MRPE is the mean of the relative prediction errors over testing curves, defined as

$$
\text { MRPE }=\frac{1}{m} \sum_{c=1}^{m} \frac{\int\left[y_{c}(t)-\hat{y}_{c}(t)\right]^{2} d t}{\int y_{c}^{2}(t) d t} .
$$

Note that the smaller the value of either MRSS or MRPE on the test data, the better the performance of the algorithm.

We compare the proposed FarMost algorithm with various function-on-function regression methods, including FdaLM (Ramsay and Silverman 2005) with $\ell_{2}$ regularization, FPCreg (Yao et al. 2005b), FAM (Müller and Yao 2008), and $\mathrm{DRFR}^{3}$ (Gervini 2015). FdaLM is available in the FDA package, while both FPCreg and FAM are available in the $\mathrm{PACE}^{4}$ package.

We repeat the experiments five-times and report the average performance. The parameters are tuned for each algorithm by cross-validation on training data. The basis functions used in both FarMost and FdaLM are Fourier series. The numbers of basis functions are empirically set to 150 .

\subsection{Influence of $q$}

It is interesting to explore how the sparsity of regression weights varies with $q$, which is used to control the degree of sparsity. Figure 1 shows the heatmaps of $W$ on weather data when $q=$ $1.0,1.2,2.0$, respectively. From Figure 1(a), we can see that when $q=1$, the values of many rows and columns in $W$ are close to zero (in black). This is due to the shrinking effect of $\ell_{2,1}$ norm imposed on $W$ and $W^{T}$. When $q$ increases from 1.0 to 1.2 , the number of zero rows or columns decreases correspondingly, which is shown in Figure 1(b). In contrast, we observe much denser regression weights in Figure $1(\mathrm{c})$, where $q=2.0$. As expected, the results verify that a small $q$ could lead to a more sparse $W$. Also, it intuitively demonstrates that the proposed mode-sparsity regularization indeed results in both row-wise and columnwise sparsity.

Next, we study the influence of $q$ on the performance of FarMost. $q$ is changed from 0.2 to 2.0 with step size 0.2 . Figures 2-3 show the performances in terms of MRPE and MRSS, respectively,

\footnotetext{
${ }^{3}$ https://people.uwm.edu/gervini/files/2016/04/DRFR_package-tbaxkq.zip.

${ }^{4}$ http://www.stat.ucdavis.edu/PACE/.
} 


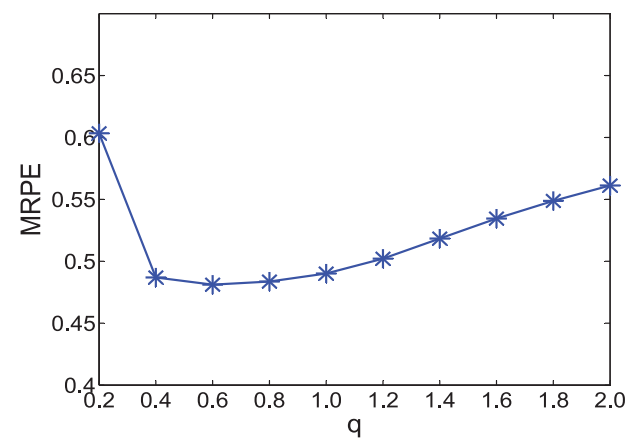

Fig. 2. MRPE vs. $q$ on weather data.

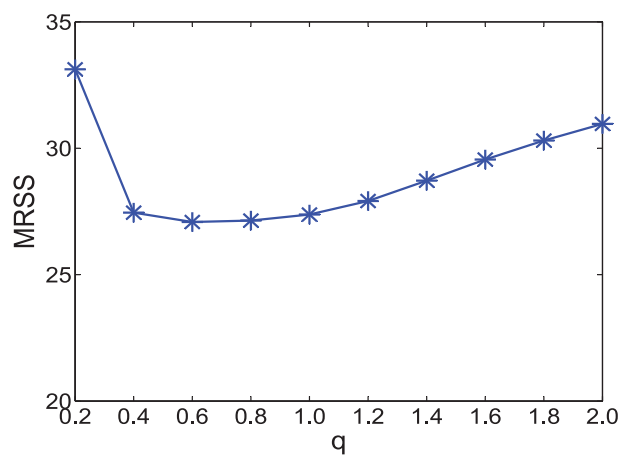

Fig. 3. MRSS vs. $q$ on weather data.

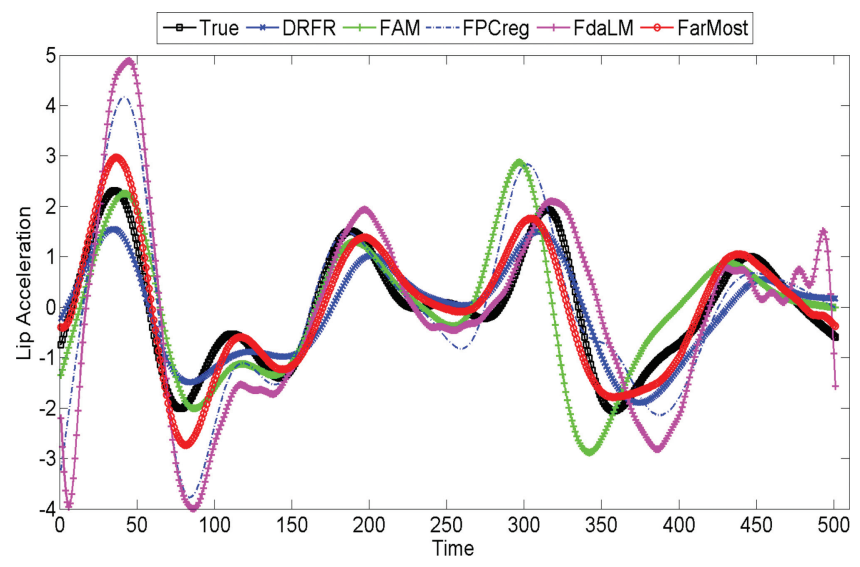

Fig. 4. Regression curves vs. grouth-truth on LipEMG.

varying with $q$ on the weather dataset. We can see from both figures that the relatively smaller $q$ $(0.4 \leq q \leq 1.2)$ usually leads to the better performance. It suggests that truncating the irrelevant basis functions for both predictor and response could improve the performance. However, a too small $q(q=0.2)$ may deteriorate the performance. This might be due to the non-convexity of objective function when $0<q<1$, which prevents the algorithm from reaching the global optimum. Therefore, we set $q=1$ in the following experiments.

\subsection{Performance Comparison}

We first examine how well the predictions of different algorithms fit the ground-truth curves on the test data. Define training rate as $\frac{n-m}{n}$, where $m$ is the number of curves for testing, and $n$ is the number of curves in total. Take the LipEMG data as an example. We use the first 29 curve pairs for model training (training rate is about 0.9), and the remaining three curve pairs for testing. Figure 4 shows the predictions of different algorithms in comparison with the ground-truth on the last response curve. We can see that most of the algorithms are basically capable of catching the trend of the ground-truth curve. However, the proposed FarMost model fits the ground-truth curve better than the others. Next, we will quantitatively measure their performance differences.

Figures 5-10 show the regression performances in terms of MRSS and MRPE, respectively, on the three datasets. In each figure, $x$-axis represents the training ratio, and $y$-axis represents the regression performances on the test data. As the standard deviations of all the methods are close to 


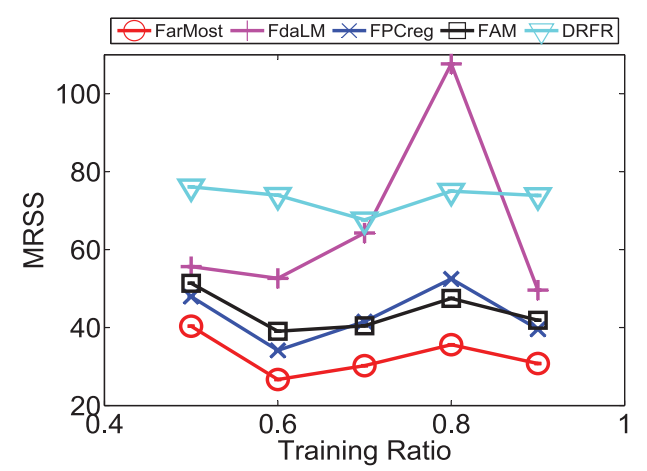

Fig. 5. MRSS on weather data.

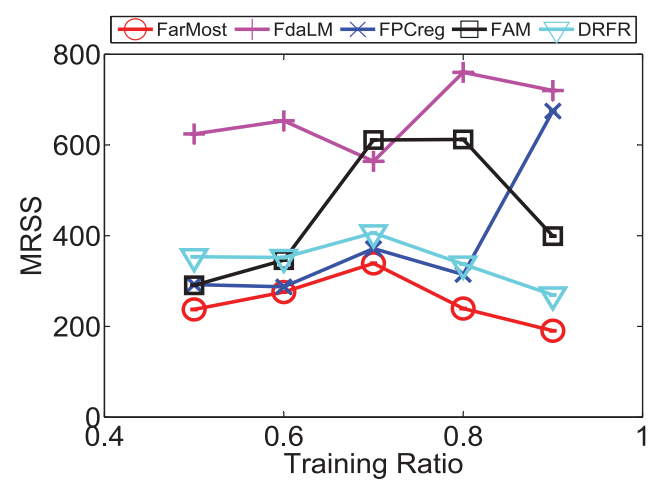

Fig. 7. MRSS on LipEMG data.

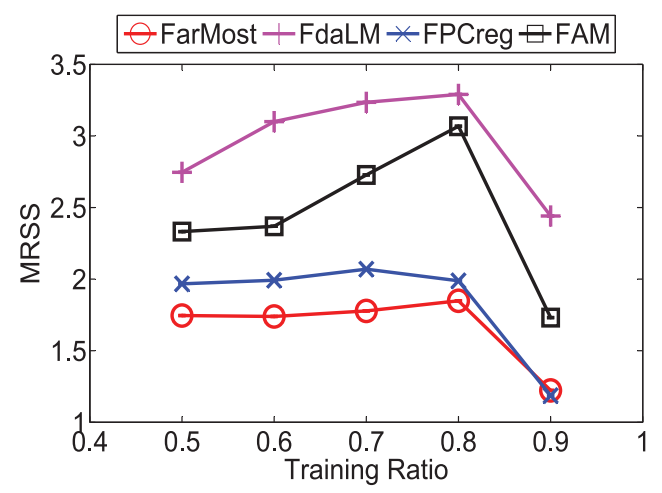

Fig. 9. MRSS on DTI2 data.

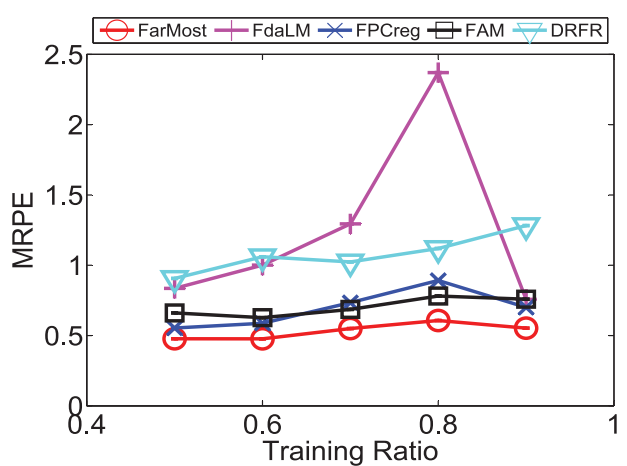

Fig. 6. MRPE on weather data.

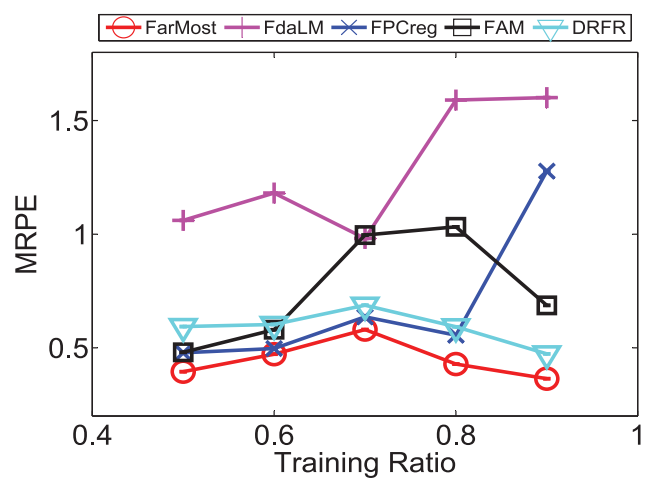

Fig. 8. MRPE on LipEMG data.

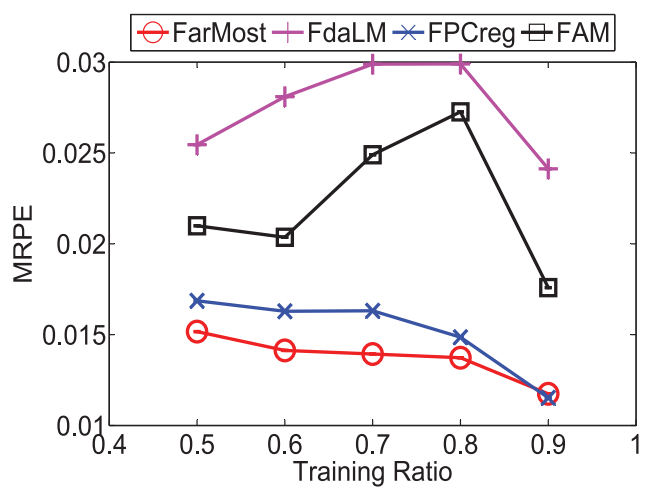

Fig. 10. MRPE on DTI2 data.

zero, we just plot the mean values on the figures. Since DRFR (Gervini 2015) requires the predictor and response to be defined on the same domain, we evaluate DRFR on the weather and LipEMG datasets only.

The results show that the proposed FarMost algorithm performs the best among all the comparison algorithms. Both FPCreg (Yao et al. 2005b) and FAM (Müller and Yao 2008) are based on FPCA that keeps only the first few principal components explaining the most variability. The results 


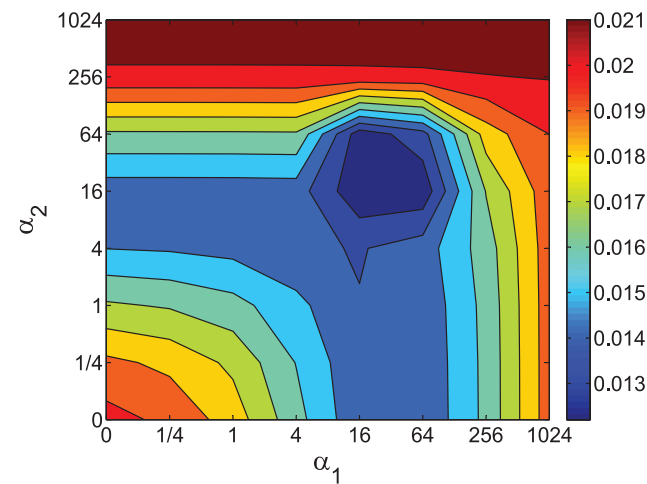

Fig. 11. The contour of MRPE varying with $\alpha_{1}$ and $\alpha_{2}$ on DTI2 ( $\log _{4}$ scale).

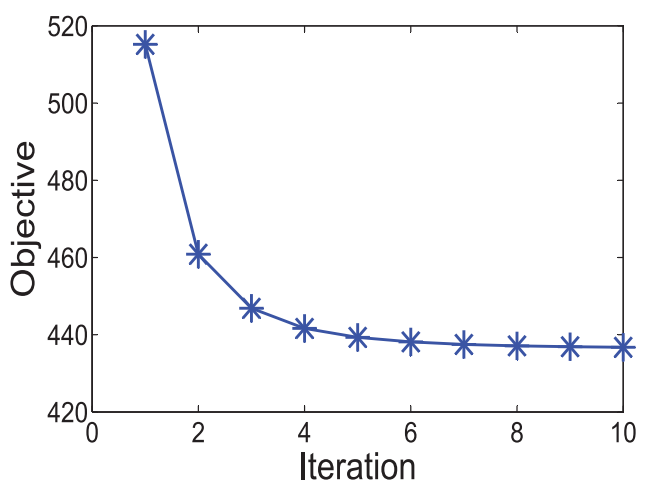

Fig. 12. Objective value varies with iterations on DTI2.

indicate that the functional principal components explaining the largest amount of variability in predictor may not necessarily be the most discriminative in predicting response. DRFR (Gervini 2015) incorporates phase synchronization as an intrinsic part of the model, thus attains better predictive power than ordinary linear regression. Figures 7-8 show that DRFR performs well on the LipEMG data since both EMG and acceleration curves have periodic peaks and valleys. We also have the consistent observations on Figure 4, where DRFR fits well with the ground-truth curve of lip acceleration. However, the performance of DRFR is limited on the weather data where there have no obvious periods in the daily temperature and precipitation profiles which are averaged over 30 years. FdaLM (Ramsay and Silverman 2005) performs worse than the other algorithms, suggesting that using only the roughness penalty ( $\ell_{2}$ regularization) may not be adequate to build a robust regression model. In contrast, FarMost uses mode-sparsity regularization to simultaneously select the informative basis functions from each mode and truncate the irrelevant ones. It improves the representation capability of the basis functions and leads to a more robust regression model. As a consequence, its generalization performance on the unseen responses can be improved.

\subsection{Parameter Sensitivity and Convergence}

We study the performance sensitivity of FarMost on the parameters $\alpha_{1}$ and $\alpha_{2}$, which are used to control the importance of mode-sparsity regularization. Figure 11 shows the contour of MRPE varying with both $\alpha_{1}$ and $\alpha_{2}$. FarMost performs worse as $\alpha_{1}$ or $\alpha_{2}$ approaches 0 when no sparsity regularization is imposed on the regression weights, while the optimal performance is achieved at $\alpha_{1}=16$ and $\alpha_{2}=16$. It suggests that imposing the mode-specific sparsity regularization on the regression model could lead to better performance. But a too large value of $\alpha_{1}$ or $\alpha_{2}$ (e.g., $\alpha_{1}, \alpha_{2} \geq$ 256) will deteriorate the performance of the FarMost algorithm, indicating that too much weight is putting on the sparsity regularization term. Nevertheless, the performance is robust over a wide range of $\alpha_{1}$ or $\alpha_{2}$ values.

Also, it is worth noticing that imposing single-way sparsity on the regression model is the special case of our method with multi-way sparsity. The results suggest that multi-way selection could improve upon single-way selection of basis functions in building a regression model.

We also study the convergence property of the proposed algorithm. Figure 12 shows the objective function value of FarMost varying with iteration. From this figure, we can see that FarMost converges fast and its objective function value becomes stable after about 10 iterations. 
Table 2. Statistics of Different Datasets

\begin{tabular}{l|ccccccc}
\hline Data set & $n$ & $x$ & $|\mathcal{S}|$ & $z$ & $|\mathcal{R}|$ & $y$ & $|\mathcal{T}|$ \\
\hline Weather & 35 & Temperature & 365 & Precipitation (prior) & 182 & Precipitation (posterior) & 183 \\
LipEMG & 32 & EMG & 501 & Acceleration (prior) & 250 & Acceleration (posterior) & 251 \\
DTI2 & 340 & FA-RCTS & 55 & FA-CCA (prior) & 46 & FA-CCA (posterior) & 47 \\
\hline
\end{tabular}

Table 3. Performance of FarMost (Tensor Version) Compared to Related Algorithms

\begin{tabular}{l|cc|cc|cc}
\hline \multirow{2}{*}{ Algorithm } & \multicolumn{2}{|c|}{ Weather } & \multicolumn{2}{c|}{ LipEMG } & \multicolumn{2}{c}{ DTI2 } \\
& MRPE & MRSS & MRPE & MRSS & MRPE & MRSS \\
\hline FarMost & $\mathbf{0 . 5 8 7} \pm \mathbf{0 . 0}$ & $\mathbf{1 0 . 2 4} \pm \mathbf{0 . 0}$ & $\mathbf{0 . 3 8 1} \pm \mathbf{0 . 0}$ & $\mathbf{9 4 . 9 6} \pm \mathbf{0 . 0}$ & $\mathbf{0 . 0 1 2} \pm \mathbf{0 . 0}$ & $\mathbf{0 . 9 1 2} \pm \mathbf{0 . 0}$ \\
FdaLM & $1.234 \pm 0.0$ & $19.04 \pm 0.0$ & $1.444 \pm 0.0$ & $337.72 \pm 0.0$ & $0.024 \pm 0.0$ & $2.062 \pm 0.0$ \\
FPCreg & $0.708 \pm 0.0$ & $12.06 \pm 0.0$ & $0.556 \pm 0.0$ & $131.75 \pm 0.0$ & $0.013 \pm 0.0$ & $1.000 \pm 0.0$ \\
FAM & $0.670 \pm 0.0$ & $11.76 \pm 0.0$ & $0.552 \pm 0.0$ & $143.92 \pm 0.0$ & $0.020 \pm 0.0$ & $1.463 \pm 0.0$ \\
\hline
\end{tabular}

\subsection{Results on Tensor Extension of FarMost}

In this subsection, we evaluate the tensor version of FarMost, which allows us to regress response on multiple predictors.

Table 2 shows the statistics of the datasets. In each dataset, we have two functional predictors, $x$ and $z$, and one response $y$. Taking the weather dataset as an example, we try to predict the posterior portion of precipitation profile when given the temperature information and the prior portion of precipitation profile.

FarMost is compared to FdaLM (Ramsay and Silverman 2005), FPCreg (Yao et al. 2005b), and FAM (Müller and Yao 2008), except for DRFR (Gervini 2015) as it requires the predictor and response to be defined on the same domain. Since the other algorithms, including FdaLM, FPCreg, and FAM can not directly work on multiple predictors, we build two independent regression models for each algorithm using the two predictors, respectively, and then average their outputs as the final results. In this experiment, we fix the training ratio as 0.9 . The significance level is set to $5 \%$.

The results are shown in Table 3. Our proposed method outperforms the other algorithms on the three datasets. The competency of FarMost is twofold. First, FarMost makes use of tensor to model not only the correlations between predictor and response, but also the correlations among multiple predictors. Second, FarMost encourages the mode-sparisty of the regression weight tensor, which improves the regression performance.

\section{CONCLUSIONS}

In this article, we propose a novel function-on-function regression model based on mode-sparsity regularization, and further extend it to a tensor version to accommodate multiple functional predictors. A simple and efficient algorithm is developed to solve the optimization problem, which works in an iterative update fashion, and converges to the global optimum. The theoretical analysis gives new insight into the consistency between the estimated function and the underlying true one. The effectiveness of the proposed approach is demonstrated on multiple functional datasets.

Note that most of current work on FDA focus on functions observed in Euclidean domains. However, the scope of functional regression goes far beyond that, with many applications yielding complex functions on non-Euclidean manifolds. In the future, we will explore the possibility of extending our model to these complex scenarios. 


\section{REFERENCES}

Peter J. Basser, Sinisa Pajevic, Carlo Pierpaoli, Jeffrey Duda, and Akram Aldroubi. 2000. In vivo fiber tractography using DT-MRI data. Magnetic Resonance in Medicine 44, 4 (2000), 625-632.

Yingying Fan, Gareth M. James, and Peter Radchenko. 2015. Functional additive regression. The Annals of Statistics 43, 5 (2015), 2296-2325.

Daniel Gervini. 2015. Dynamic retrospective regression for functional data. Technometrics 57, 1 (2015), 26-34.

Jeff Goldsmith, Jennifer Bobb, Ciprian M. Crainiceanu, Brian Caffo, and Daniel Reich. 2011. Penalized functional regression. Journal of Computational and Graphical Statistics 20, 4 (2011), 830-851.

Gene H. Golub and Charles F. Van Loan. 1996. Matrix Computations (3rd ed.). Johns Hopkins University Press.

Jaroslaw Harezlak, Brent A. Coull, Nan M. Laird, Shannon R. Magari, and David Christiani. 2007. Penalized solutions to functional regression problems. Computational Statistics \& Data Analysis 51, 10 (2007), 4911-4925.

Andrada E. Ivanescu, Ana-Maria Staicu, Fabian Scheipl, and Sonja Greven. 2015. Penalized function-on-function regression. Computational Statistics 30, 2 (2015), 539-568.

Gareth M. James, Jing Wang, and Ji Zhu. 2009. Functional linear regression that's interpretable. The Annals of Statistics 37, 5A (2009), 2083-2108.

Hachem Kadri, Emmanuel Duflos, Philippe Preux, Stéphane Canu, and Manuel Davy. 2010. Nonlinear functional regression: A functional RKHS approach. In AISTATS. 374-380.

Hachem Kadri, Emmanuel Duflos, Philippe Preux, Stéphane Canu, Alain Rakotomamonjy, and Julien Audiffren. 2016. Operator-valued kernels for learning from functional response data. Journal of Machine Learning Research 17 (2016), 20:1-20:54

Tamara G. Kolda and Brett W. Bader. 2009. Tensor decompositions and applications. SIAM Review 51, 3 (2009), 455-500.

John M. Koomen, Lichen N. Shih, Kevin R. Coombes, Donghui Li, Lian-chun Xiao, Isaiah J. Fidler, James L. Abbruzzese, and Ryuji Kobayashi. 2005. Plasma protein profiling for diagnosis of pancreatic cancer reveals the presence of host response proteins. Clinical Cancer Research 11, 3 (2005), 1110-1118.

Eun R. Lee and Byeong U. Park. 2012. Sparse estimation in functional linear regression. fournal of Multivariate Analysis 105, 1 (2012), 1-17.

Daniel J. Levitin, Regina L. Nuzzo, Bradley W. Vines, and J. O. Ramsay. 2007. Introduction to functional data analysis. Canadian Psychology 48, 3 (2007), 135-155.

Mark J. Meyer, Brent A. Coull, Francesco Versace, Paul Cinciripini, and Jeffrey S. Morris. 2015. Bayesian function-onfunction regression for multilevel functional data. Biometrics 71, 3 (2015), 563-574.

Jeffrey S. Morris. 2015. Functional regression. Annual Review of Statistics and Its Application 2, 1 (2015), 321-359.

Hans-Georg Müller and Fang Yao. 2008. Functional additive models. Journal of the American Statistical Association 103, 484 (2008), 1534-1544.

Feiping Nie, Heng Huang, and Chris H. Q. Ding. 2012. Low-rank matrix recovery via efficient Schatten p-norm minimization. In $A A A I$.

Jorge Nocedal and Stephen Wright. 2006. Numerical Optimization (2nd ed.). Springer-Verlag, New York.

Junier B. Oliva, Willie Neiswanger, Barnabás Póczos, Eric P. Xing, Hy Trac, Shirley Ho, and Jeff G. Schneider. 2015. Fast function to function regression. In AISTATS.

J. O. Ramsay and B. W. Silverman. 2002. Applied Functional Data Analysis (1st ed.). Springer-Verlag, New York.

J. O. Ramsay and B. W. Silverman. 2005. Functional Data Analysis (2nd ed.). Springer-Verlag, New York.

Fabian Scheipl, Ana-Maria Staicu, and Sonja Greven. 2015. Functional additive mixed models. Journal of Computational and Graphical Statistics 24, 2 (2015), 477-501.

G. A. Watson. 1992. Characterization of the subdifferential of some matrix norms. Linear Algebra and Its Applications170 (1992), 33-45.

Pei Yang and Jingrui He. 2016. Functional regression with mode-sparsity constraint. In ICDM. 1311-1316.

Fang Yao, Hans-Georg Müller, and Jane-Ling Wang. 2005a. Functional data analysis for sparse longitudinal data. fournal of the American Statistical Association 100, 470 (2005), 577-590.

Fang Yao, Hans-Georg Müller, and Jane-Ling Wang. 2005b. Functional linear regression analysis for longitudinal data. The Annals of Statistics 33, 6 (2005), 2873-2903.

Jin Yu, S. V. N. Vishwanathan, Simon Günter, and Nicol N. Schraudolph. 2010. A quasi-Newton approach to nonsmooth convex optimization problems in machine learning. fournal of Machine Learning Research 11 (2010), 1145-1200.

Ming Yuan and T. T. Cai. 2010. A reproducing kernel Hilbert space approach to functional linear regression. The Annals of Statistics 38, 6 (2010), 3412-3444.

Daowen Zhang, Xihong Lin, and MaryFran Sowers. 2007. Two-stage functional mixed models for evaluating the effect of longitudinal covariate profiles on a scalar outcome. Biometrics 63, 2 (2007), 351-362.

Yihong Zhao, Huaihou Chen, and R. Todd Ogden. 2015. Wavelet-based weighted LASSO and screening approaches in functional linear regression. Journal of Computational and Graphical Statistics 24, 3 (2015), 655-675. 
Yihong Zhao, R. Todd Ogden, and Philip T. Reiss. 2012. Wavelet-based LASSO in functional linear regression. fournal of Computational and Graphical Statistics 21, 3 (2012), 600-617.

Hongxiao Zhu and Dennis D. Cox. 2009. A functional generalized linear model with curve selection in cervical pre-cancer diagnosis using fluorescence spectroscopy. Lecture Notes-Monograph Series 57 (2009), 173-189.

Received November 2016; revised October 2017; accepted December 2017 